\title{
A New Type Method for the Structured Variational Inequalities Problem
}

\author{
Chengjiang Yin \\ Linyi University at Feixian \\ Feixian, Shandong, P.R.China
}

\begin{abstract}
In this paper, we present an algorithm for solving the structured variational inequality problem, and prove the global convergence of the new method without carrying out any line search technique, and the global $\mathbf{R}$-convergence rate are also given under the suitable conditions.
\end{abstract}

Keywords-structured variational inequality problem; algorithm; globally convergent; R-linear convergent

\section{INTRODUCTION}

Leting mappings $f: X \rightarrow R^{n}, g: Y \rightarrow R^{m}$, the structured variational inequality problem with linear constraint is to find vector $u^{*} \in \Omega$ such that

$$
\left(u-u^{*}\right)^{T} T\left(u^{*}\right) \geq 0, u \in \Omega
$$

where $u=\left(\begin{array}{l}x \\ y\end{array}\right), T(u)=\left(\begin{array}{l}f(x) \\ g(y)\end{array}\right), \Omega=\{(x, y) \mid x \in X, y \in Y, A x+$ $B y=b\}, X \subseteq R^{n}, Y \subseteq R^{m}$ are given nonempty closed convex sets, $f, g$ are given continuous monotone operators, $A \in R^{r \times n}, B \in R^{r \times m}, b \in R^{r}$. We denote the solution set of the VI by $\Omega^{*}$, and assume that it is nonempty throughout this paper.

By attaching a Lagrange multiplier vector $\lambda \in R^{r}$ to the linear constraints $A x+B y=b$,(1.1)can be equivalently transformed into the following compact form, denoted by VI: Find $w^{*} \in W$ such that

$$
\begin{gathered}
\left(w-w^{*}\right)^{T} Q\left(w^{*}\right) \geq 0, w \in W \\
\text { where } w=\left(\begin{array}{c}
x \\
y \\
z
\end{array}\right), Q(w)=\left(\begin{array}{c}
f(x)+A^{T} \lambda \\
g(y)+B^{T} \lambda \\
A x+b y-b
\end{array}\right), W=X \times Y \times R^{r} \text {, and }
\end{gathered}
$$

the solution set of (1.2) is denoted by $W^{*}$ which is always assumed to be nonempty.

This problem has important applications in many fields, such as network economics, traffic assignment, game theoretic problems, etc. For example, Nagurney et al. ([1]) developed a variational inequality based supply chain network equilibrium model consisting of three tiers of decisionmakers in the network. They established some governing equilibrium conditions based on the optimality conditions of the decision-makers along with the market equilibrium conditions. In recent years, many methods have been proposed to solve the VI ([2-8]). The alternating direction method $(\mathrm{ADM})$ is a powerful method for solving the structured problem (1.2), since it decompose the original problems into a series subproblesm with lower scale, which was originally proposed by Gabay and Mercier ([5]) and Gabay ( [6] ). Ye and Yuan [7] proposed a new descent method for VI by adding an additional projection step to the above ADM. Han ([8]) proposed a modified alternating direction method for variational inequalities with linear constraints. At each iteration, the method only makes an orthogonal projection to simple set and some function evaluations. Motivated by [7, 8], we present a new algorithm for the structured variational inequality problem, and prove the global convergence of the new method without carrying out any line search technique. Furthermore, we also show that this method is global $R$ - linear convergent under the suitable conditions.

Some notations used in this paper are in order. The vectors considered in this paper are all taken in Euclidean space equipped with the standard inner product, which is denoted by $R^{n}$. We let $\|\not\|$ and \|\|$_{1}$ respectively denote the usual Euclidean 2-norm and 1-norm of vectors in $R^{n}$. The transpose of matrix $M$ (vector $x$ ) be denoted by $M^{T}\left(x^{T}\right)$.

\section{PRELIMINARIES}

In this section, we first give the following definition of projection operator and some relate properties ([9]). For nonempty closed convex set $\Omega \subset R^{n}$ and any vector $x \in R^{n}$, the orthogonal projection of $x$ onto $\Omega$ is denoted by $P_{\Omega}(x)$.

Lemma 2.1 For any $u \in R^{n}, v \in \Omega$, then $\left\langle P_{\Omega}(u)-u, v-\right.$ $\left.P_{\Omega}(u)\right\rangle \geq 0$.

For (1.2), $\beta>0$ is constant,

$$
\begin{aligned}
e(w, \beta) & =w-P_{w}[w-\beta Q(w)] \\
& =\left(\begin{array}{l}
e_{1}(w, \beta) \\
e_{2}(w, \beta) \\
e_{3}(w, \beta)
\end{array}\right)=\left(\begin{array}{l}
x-P_{X}\left[x-\beta\left(f(x)+A^{T} \lambda\right)\right] \\
y-P_{Y}\left[y-\beta\left(g(y)+B^{T} \lambda\right)\right] \\
\beta(A x+B y-b)
\end{array}\right)
\end{aligned}
$$

is called projection-type residual function, and let $r(\omega):=\|e(\omega)\|$. The following conclusion provides the 
relationship between the solution set of (1.2) and that of projection-type residual function ([10]).

Lemma 2.2 $\omega$ is a solution of (1.2) if and only if $r(\omega)=0$.

To establish theoretical analysis of the following algorithm, we also need the following definition.

Definition 2.1 The mapping $f: R^{n} \rightarrow R^{m}$ is said to be cocoercive with modulus $\mu>0$ is

$$
\langle f(x)-f(y), x-y\rangle \geq \mu\|f(x)-f(y)\|^{2}, \quad \forall x, y \in R^{n} .
$$

Definition 2.2 The mapping $f: R^{n} \rightarrow R^{m}$ is said to be strongly monotone if there is constant $\mu>0$ such that

$$
\langle f(x)-f(y), x-y\rangle \geq \mu\|x-y\|^{2}, \quad \forall x, y \in R^{n} .
$$

Obviously, suppose that $f$ is strongly monotone with positive constant $\mu$, and is Lipschitz continuous with positive constant $L>0$. Then the $f$ is co-coercive, i.e., for any $x, y \in R^{n}$, we have

$$
\begin{aligned}
\langle f(x)-f(y), x-y\rangle & \geq \mu\|x-y\|^{2}=\frac{\mu}{L^{2}}(L\|x-y\|)^{2} \\
& \geq \frac{\mu}{L^{2}}\|f(x)-f(y)\|^{2} .
\end{aligned}
$$

\section{Algorithm AND CONVERGENCE}

In this following, we formally state our algorithm.

\section{Algorithm 3.1}

Step1. Take $\varepsilon>0,0<\beta<2 \min \left\{\frac{\sqrt{\mu_{1}}}{\sqrt{n}}, \frac{\sqrt{\mu_{2}}}{\sqrt{m}}, \frac{\sqrt{\mu_{3}}}{\sqrt{r}}\right\}$, where the positive constants $\mu_{1}, \mu_{2}, \mu_{3}$ are defined in the following Theorem 3.1, and take initial point $x^{0} \in R^{n}$. Set $k=0$;

Step2. Compute

$$
\omega^{k+1}=P_{W}\left[\omega^{k}-\beta Q\left(\omega^{k}\right)\right]=\left(\begin{array}{c}
P_{X}\left[x^{k}-\beta\left(f\left(x^{k}\right)+A^{T} \lambda^{k}\right)\right] \\
P_{Y}\left[y^{k}-\beta\left(g\left(y^{k}\right)+B^{T} \lambda^{k}\right)\right] \\
\lambda^{k}-\beta\left(A x^{k}+B y^{k}-b\right)
\end{array}\right)
$$

Step3. If $\left\|\omega^{k+1}-\omega^{k}\right\| \leq \varepsilon$ stop, otherwise, go to Step 2 with $k:=k+1$.

Remark The algorithm is based on problem (1.2). Obviously, if $\omega^{k+1}=\omega^{k}$, combining Lemma 2.2, then $\omega^{k}$ is a solution of (1.2), and so is also (1.1). In the following theoretical analysis, we assume that Algorithm 3.1 generates an infinite sequence.

Lemma 3.1 Suppose that the matrix $\bar{M}$ is positive semidefinite, and $\mu_{\max } \neq 0$ is a maximum eigenvalue of $\bar{M}$, we have
$y^{T} \bar{M} y \geq\left(1 / \mu_{\max }\right)\|\bar{M} y\|^{2}\left(\forall y \in R^{l}\right)$, where $\bar{M}=\left(\begin{array}{ccc}0 & 0 & A^{T} \\ 0 & 0 & B^{T} \\ A & B & 0\end{array}\right)$.

Proof: Since the matrix $\bar{M}$ is positive semi-definite, then there exists an orthogonal matrix $P$ such that $P \bar{M} P^{\cdot}=\operatorname{diag}\left(\sigma_{1}, \sigma_{2}, \cdots, \sigma_{s}, 0, \cdots, 0\right)$, where $\sigma_{1} \geq \sigma_{2} \geq \cdots \geq \sigma_{s}>0$.

Set $\xi=P y$, then

$$
\begin{aligned}
y^{\bullet} \bar{M} y & =y^{\bullet} P^{\bullet} \operatorname{diag}\left(\sigma_{1}, \sigma_{2}, \cdots, \sigma_{s}, 0 \cdots, 0\right) P y \\
& \geq\left(1 / \mu_{\max }\right)\left(\sigma_{1}^{2} \xi_{1}^{2}+\sigma_{2}^{2} \xi_{2}^{2}+\cdots+\sigma_{s}^{2} \xi_{s}^{2}\right) \\
& =\left(1 / \mu_{\max }\right) y^{\cdot} P^{\cdot} \operatorname{diag}\left(\sigma_{1}^{2}, \sigma_{2}^{2}, \cdots, \sigma_{s}^{2}, 0 \cdots, 0\right) P y \\
& =\left(1 / \mu_{\max }\right)\|\bar{M} y\|^{2} .
\end{aligned}
$$

Theorem 3.1 Suppose that $f, g$ are co-coercive with positive constants $\mu_{1}, \mu_{2}$, respectively, and the matrix $\bar{M}$ is positive semi-definite.

Then the sequence $\left\{\omega^{k}\right\}$ converges globally to a solution of (1.1).

Proof: By Lemma 2.1, we have

$$
\left\langle\beta Q\left(\omega^{k}\right)+\left[\omega^{k+1}-\omega^{k}\right], \omega-\omega^{k+1}\right\rangle \geq 0, \forall \omega \in W .
$$

We take $\omega^{*} \in W^{*}$, then

$$
\left\langle\beta Q\left(\omega^{*}\right), \omega-\omega^{*}\right\rangle \geq 0, \forall \omega \in W .
$$

Let $\omega=\omega^{*}$ in (3.2), take $\omega=\omega^{k+1}$ in (3.3), adding these two inequalities yields

$$
\begin{aligned}
0 \leq & \left\langle\beta\left[Q\left(\omega^{k}\right)-Q\left(\omega^{*}\right)\right]+\omega^{k+1}-\omega^{k}, \omega^{*}-\omega^{k+1}\right\rangle \\
= & \left\langle\beta\left[Q\left(\omega^{k}\right)-Q\left(\omega^{*}\right)\right], \omega^{*}-\omega^{k+1}\right\rangle+\left\langle\omega^{k+1}-\omega^{k}, \omega^{*}-\omega^{k+1}\right\rangle \\
= & \left\langle-\beta\left[Q\left(\omega^{k}\right)-Q\left(\omega^{*}\right)\right], \omega^{k}-\omega^{*}+\omega^{k+1}-\omega^{k}\right\rangle+ \\
& \left\langle\omega^{k+1}-\omega^{k}, \omega^{*}-\omega^{k+1}\right\rangle \\
= & \left\langle-\beta\left[Q\left(\omega^{k}\right)-Q\left(\omega^{*}\right)\right], \omega^{k}-\omega^{*}\right\rangle-\left\langle\beta\left[Q\left(\omega^{k}\right)-Q\left(\omega^{*}\right)\right], \omega^{k+1}-\omega^{k}\right\rangle \\
& +\left\langle\omega^{k+1}-\omega^{k}, \omega^{*}-\omega^{k+1}\right\rangle \\
& -\beta\left(\begin{array}{l}
\left(f\left(x^{k}\right)-f\left(x^{*}\right)\right)+A^{T}\left(\lambda^{k}-\lambda^{*}\right) \\
\left(g\left(y^{k}\right)-g\left(y^{*}\right)\right)+B^{T}\left(\lambda^{k}-\lambda^{*}\right) \\
A\left(x^{k}-x^{*}\right)+B\left(y^{k}-y^{*}\right)
\end{array}\right)\left(\begin{array}{c}
x^{k}-x^{*} \\
y^{k}-y^{*} \\
\lambda^{k}-\lambda^{*}
\end{array}\right) \\
& -\left\langle\beta\left[Q\left(\omega^{k}\right)-Q\left(\omega^{*}\right)\right], \omega^{k+1}-\omega^{k}\right\rangle+\left\langle\omega^{k+1}-\omega^{k}, \omega^{*}-\omega^{k+1}\right\rangle \\
= & -\beta\left[\left(f\left(x^{k}\right)-f\left(x^{*}\right)\right)^{T}\left(x^{k}-x^{*}\right)+\left(g\left(y^{k}\right)-g\left(y^{*}\right)\right)^{T}\left(y^{k}-y^{*}\right)\right] \\
& -\beta\left[\left(\lambda^{k}-\lambda^{*}\right)^{T} A\left(x^{k}-x^{*}\right)+\left(x^{k}-x^{*}\right)^{T} A^{T}\left(\lambda^{k}-\lambda^{*}\right)+\left(\lambda^{k}-\lambda^{*}\right)^{T} B\left(y^{k}-y^{*}\right)\right. \\
& \left.+\left(y^{k}-y^{*}\right)^{T} B^{T}\left(\lambda^{k}-\lambda^{*}\right)\right] \\
& -\left\langle\beta\left[Q\left(\omega^{k}\right)-Q\left(\omega^{*}\right)\right], \omega^{k+1}-\omega^{k}\right\rangle+\left\langle\omega^{k+1}-\omega^{k}, \omega^{*}-\omega^{k+1}\right\rangle \\
\leq & -\beta\left[\mu \mu_{1}\left\|f\left(x^{k}\right)-f\left(x^{*}\right)\right\|^{2}+\mu_{2}\left\|g\left(y^{k}\right)-g\left(y^{*}\right)\right\|^{2}+\mu_{3} \| \begin{array}{c}
A^{T}\left(\lambda^{k}-\lambda^{*}\right) \\
B^{T}\left(\lambda^{k}-\lambda^{*}\right) \\
A\left(x^{k}-x^{*}\right)+B\left(y^{k}-y^{*}\right)
\end{array}\right) \|^{2} \\
& -\left\langle\beta\left[Q\left(\omega^{k}\right)-Q\left(\omega^{*}\right)\right], \omega^{k+1}-\omega^{k}\right\rangle+\left\langle\omega^{k+1}-\omega^{k}, \omega^{*}-\omega^{k+1}\right\rangle
\end{aligned}
$$




$$
\begin{aligned}
& \leq-\beta\left[\mu_{1}\left\|f\left(x^{k}\right)-f\left(x^{*}\right)\right\|^{2}+\mu_{2}\left\|g\left(y^{k}\right)-g\left(y^{*}\right)\right\|^{2}+\mu_{3}\left\|A\left(x^{k}-x^{*}\right)+B\left(y^{k}-y^{*}\right)\right\|^{2}\right] \\
& -\left\langle\beta\left[Q\left(\omega^{k}\right)-Q\left(\omega^{*}\right)\right], \omega^{k+1}-\omega^{k}\right\rangle+\left\langle\omega^{k+1}-\omega^{k}, \omega^{*}-\omega^{k+1}\right\rangle \\
& \leq-\beta\left[\sqrt{\mu_{1}}\left\|f\left(x^{k}\right)-f\left(x^{*}\right)\right\|+\sqrt{\mu_{2}}\left\|g\left(y^{k}\right)-g\left(y^{*}\right)\right\|+\sqrt{\mu_{3}}\left\|A\left(x^{k}-x^{*}\right)+B\left(y^{k}-y^{*}\right)\right\|\right]^{2} \\
& -\left\langle\beta\left[Q\left(\omega^{k}\right)-Q\left(\omega^{*}\right)\right], \omega^{k+1}-\omega^{k}\right\rangle+\left\langle\omega^{k+1}-\omega^{k}, \omega^{*}-\omega^{k+1}\right\rangle \\
& \leq-\beta\left[\frac{\sqrt{\mu_{1}}}{\sqrt{n}}\left\|f\left(x^{k}\right)-f\left(x^{*}\right)\right\|_{1}+\frac{\sqrt{\mu_{2}}}{\sqrt{m}}\left\|g\left(y^{k}\right)-g\left(y^{*}\right)\right\|_{1}+\frac{\sqrt{\mu_{3}}}{\sqrt{r}}\left\|A\left(x^{k}-x^{*}\right)+B\left(y^{k}-y^{*}\right)\right\|_{1}\right]^{2} \\
& -\left\langle\beta\left[Q\left(\omega^{k}\right)-Q\left(\omega^{*}\right)\right], \omega^{k+1}-\omega^{k}\right\rangle+\left\langle\omega^{k+1}-\omega^{k}, \omega^{*}-\omega^{k+1}\right\rangle \\
& \left.\leq-\beta \mu\left[\left\|\left(\begin{array}{c}
f\left(x^{k}\right)-f\left(x^{*}\right) \\
g\left(y^{k}\right)-g\left(y^{*}\right) \\
\left(A x^{k}+B y^{k}-b\right)-\left(A x^{*}+B y^{*}-b\right)
\end{array}\right)\right\|_{1}\right]\right]^{2}-\left\langle\beta\left[Q\left(\omega^{k}\right)-Q\left(\omega^{*}\right)\right], \omega^{k+1}-\omega^{k}\right\rangle \\
& +\left\langle\omega^{k+1}-\omega^{k}, \omega^{*}-\omega^{k+1}\right\rangle \\
& \leq-\beta \mu\left[\left\|\left(\begin{array}{c}
f\left(x^{k}\right)-f\left(x^{*}\right) \\
g\left(y^{k}\right)-g\left(y^{*}\right) \\
\left(A x^{k}+B y^{k}-b\right)-\left(A x^{*}+B y^{*}-b\right)
\end{array}\right)\right\|\right]^{2}-\left\langle\beta\left[Q\left(\omega^{k}\right)-Q\left(\omega^{*}\right)\right], \omega^{k+1}-\omega^{k}\right\rangle \\
& +\left\langle\omega^{k+1}-\omega^{k}, \omega^{*}-\omega^{k+1}\right\rangle \\
& \leq-\beta \mu\left[\left\|\left(Q\left(\omega^{k}\right)-Q\left(\omega^{*}\right)\right)\right\|\right]^{2}+\left\langle\beta\left[Q\left(\omega^{k}\right)-Q\left(\omega^{*}\right)\right], \omega^{k}-\omega^{k+1}\right\rangle \\
& +\left\langle\omega^{k+1}-\omega^{k}, \omega^{*}-\omega^{k+1}\right\rangle \\
& \leq-\beta \mu\left[\left\|Q\left(\omega^{k}\right)-Q\left(\omega^{*}\right)\right\|\right]^{2}+\beta\left\|Q\left(\omega^{k}\right)-Q\left(\omega^{*}\right)\right\|\left\|\omega^{k+1}-\omega^{k}\right\| \\
& +\left\langle\omega^{k+1}-\omega^{k}, \omega^{*}-\omega^{k+1}\right\rangle \\
& \leq-\beta \mu\left\|Q\left(\omega^{k}\right)-Q\left(\omega^{*}\right)\right\|^{2}+\beta \mu\left\|Q\left(\omega^{k}\right)-Q\left(\omega^{*}\right)\right\|^{2}+\frac{\beta}{4 \mu}\left\|\omega^{k+1}-\omega^{k}\right\|^{2} \\
& +\left\langle\omega^{k+1}-\omega^{k}, \omega^{*}-\omega^{k+1}\right\rangle \\
& =\frac{\beta}{4 \mu}\left\|\omega^{k+1}-\omega^{k}\right\|^{2}-\frac{1}{2}\left\|\omega^{*}-\omega^{k+1}\right\|^{2}+\frac{1}{2}\left\|\omega^{*}-\omega^{k}\right\|^{2}-\frac{1}{2}\left\|\omega^{k+1}-\omega^{k}\right\|^{2} \\
& =\frac{1}{2}\left(\frac{\beta}{2 \mu}-1\right)\left\|\omega^{k+1}-\omega^{k}\right\|^{2}-\frac{1}{2}\left\|\omega^{*}-\omega^{k+1}\right\|^{2}+\frac{1}{2}\left\|\omega^{*}-\omega^{k}\right\|^{2},
\end{aligned}
$$

where the second inequality follows from the fact that $f, g$ are co-coercive with positive constants $\mu_{1}, \mu_{2}$ and Lemma 3.1; the fourth inequality is obtained by

$$
a^{2}+b^{2}+c^{2} \leq(a+b+c)^{2} \quad(a \geq 0, b \geq 0, c \geq 0),
$$

the fifth inequality and the seventh inequality follow from the fact that

$$
\|x\| \leq\|x\|_{1} \leq \sqrt{n}\|x\|, \forall x \in R^{n}
$$

and the sixth inequality is true by letting $\mu=\min$ $\left\{\frac{\sqrt{\mu_{1}}}{\sqrt{n}}, \frac{\sqrt{\mu_{2}}}{\sqrt{m}}, \frac{\sqrt{\mu_{3}}}{\sqrt{r}}\right\}$, the ninth inequality follows from the Cauchy-Schwarz inequality. Thus, we have

$$
\left(\frac{\beta}{2 \mu}-1\right)\left\|\omega^{k+1}-\omega^{k}\right\|^{2}-\left\|\omega^{*}-\omega^{k+1}\right\|^{2}+\left\|\omega^{*}-\omega^{k}\right\|^{2} \geq 0 .
$$

Combining this with $0<\beta<2 u$, we have

$$
\left\|\omega^{k+1}-\omega^{k}\right\|^{2} \leq \frac{2 u-\beta}{2 u}\left\|\omega^{*}-\omega^{k}\right\|^{2}-\frac{2 u-\beta}{2 u}\left\|\omega^{*}-\omega^{k+1}\right\|^{2}
$$

i.e.,

$$
r\left(\omega^{k}\right)^{2} \leq \frac{2 u-\beta}{2 u}\left\|\omega^{*}-\omega^{k}\right\|^{2}-\frac{2 u-\beta}{2 u}\left\|\omega^{*}-\omega^{k+1}\right\|^{2} .
$$

by (3.4), we conclude that the nonnegative sequence $\left\{\left\|\omega^{k}-\omega^{*}\right\|_{\}}\right.$is strictly decreasing and convergent. Thus, we have $r\left(x^{k}\right)$ converges to 0 by (3.5). We also obtain that the sequence $\left\{\left\|\omega^{k}-\omega^{*}\right\|\right\}$ is bounded since it is convergent, and so is $\left\{\omega^{k}\right\}$. Let $\left\{\omega^{k_{i}}\right\}$ be a subsequence of $\left\{\omega^{k}\right\}$ and converges to $\bar{\omega}$, since $r(\omega)$ is continuous, we have $r(\bar{\omega})=0$, i.e., $\bar{\omega}$ is a solution of (1.1).

On the other hand, we suppose that $\omega$ is also a accumulation point of $\left\{\omega^{k}\right\}$, and let $\left\{\omega^{k_{j}}\right\}$ be a subsequence of $\left\{\omega^{k}\right\}$ and converges to $\omega$. For any ${ }^{k_{j}}$, there exists ${ }^{i}$ such that $k_{i}<k_{j}$, by (3.4), we obtain that $\left\|\omega^{k_{j}}-\hat{\omega}\right\|^{2} \leq\left\|\omega^{k_{i}}-\hat{\omega}\right\|^{2} \rightarrow 0$ as $k_{i} \rightarrow \infty$. Using (3.4) again, we can also obtain $\left\|\omega^{k_{j}}-\omega^{k_{i}}\right\| \rightarrow 0$. Thus, we have

$$
\|\hat{\omega}-\bar{\omega}\| \leq\left\|\omega^{k_{i}}-\bar{\omega}\right\|+\left\|\omega^{k_{j}}-\hat{\omega}\right\|+\left\|\omega^{k_{j}}-\omega^{k_{i}}\right\| \rightarrow 0 \text { as } k_{i} \rightarrow \infty,
$$

i.e., $\hat{\omega}=\bar{\omega}$. Thus, the sequence $\left\{\omega^{k}\right\}$ converges globally to a solution of (1.1).

To establish the $R$ - linear convergence rate of Algorithm3.1, we also need the following conclusion which is crucial to convergence rate of algorithm.

Lemma 3.2 Suppose that $f, g$ are strongly monotone with positive constants $\mu_{1}, \mu_{2}$, and are Lipschitz continuous with positive constants $L_{1}>0, L_{2}>0$, respectively, the matrix $\left(\begin{array}{l}A^{T} \\ B^{T}\end{array}\right)$ has full-column rank. Then for any $\omega \in R^{n+m+r}$, there exists a solution $\omega^{*}$ of (1.1) such that

$$
\left\|\omega-\omega^{*}\right\| \leq \max \left\{u_{1}^{-1}, u_{2}^{-1}, v_{3}^{-1}\right\} \frac{1}{\beta}\{1+\beta L\} r(\omega) .
$$

Proof: Since $\omega-e(\omega)=P_{W}[\omega-\beta Q(\omega)] \in W$, by (1.1),

$$
\beta\left(\omega-e(\omega)-\omega^{*}\right)^{T} Q\left(\omega^{*}\right) \geq 0 .
$$

Combining $\omega^{*} \in W^{*}$ with Lemma 2.1, we have

$$
\left\langle\omega^{*}-P_{W}[\omega-\beta Q(\omega)], P_{W}[\omega-\beta Q(\omega)]-[\omega-\beta Q(\omega)]\right\rangle \geq 0 .
$$

Substituting $P_{W}[\omega-\beta Q(\omega)]$ in (3.8) by $\omega-e(\omega)$ yields

$$
\left(\omega-\omega^{*}-e(\omega)\right)^{T}[e(\omega)-\beta Q(\omega)] \geq 0 .
$$

Adding (3.7) and (3.9), one has

$$
\left[\left(\omega-\omega^{*}\right)-e(\omega)\right]^{T}\left[e(\omega)+\beta\left(Q\left(\omega^{*}\right)-Q(\omega)\right)\right] \geq 0,
$$


i.e.,

$\left(\omega-\omega^{*}\right)^{T} \beta\left[Q\left(\omega^{*}\right)-Q(\omega)\right]+e(\omega)^{T}\left[\left(\omega-\omega^{*}\right)-\beta\left(Q\left(\omega^{*}\right)-Q(\omega)\right)\right]$

$-e(\omega)^{T} e(\omega) \geq 0$.

Combining this with Definition 2.2, a direct computation yields

$$
\begin{aligned}
& \left\|\omega-\omega^{*}\right\|^{2}=\left\|x-x^{*}\right\|^{2}+\left\|y-y^{*}\right\|^{2}+\left\|\lambda-\lambda^{*}\right\|^{2} \\
& \leq \max \left\{u_{1}^{-1}, u_{2}^{-1}, v_{3}^{-1}\right\}\left[\left(f(x)-f\left(x^{*}\right)\right)^{T}\left(x-x^{*}\right)+\left(g(y)-g\left(y^{*}\right)\right)^{T}\left(y-y^{*}\right)\right. \\
& \left.+\left(\omega-\omega^{*}\right)^{T} \bar{M}\left(\omega-\omega^{*}\right)\right] \\
& =\max \left\{u_{1}^{-1}, u_{2}^{-1}, v_{3}^{-1}\right\}\left(\begin{array}{l}
\left(f(x)-f\left(x^{*}\right)\right)+A^{T}\left(\lambda-\lambda^{*}\right) \\
\left(g(y)-g\left(y^{*}\right)\right)+B^{T}\left(\lambda-\lambda^{*}\right) \\
A\left(x-x^{*}\right)+B\left(y-y^{*}\right)
\end{array}\right)\left(\begin{array}{l}
x-x^{*} \\
y-y^{*} \\
\lambda-\lambda^{*}
\end{array}\right) \\
& =\max \left\{u_{1}^{-1}, u_{2}^{-1}, v_{3}^{-1}\right\}\left(\begin{array}{l}
\left(f(x)+A^{T} \lambda\right)-\left(f\left(x^{*}\right)+A^{T} \lambda^{*}\right) \\
\left(g(y)+B^{T} \lambda\right)-\left(g\left(y^{*}\right)+B^{T} \lambda^{*}\right) \\
(A x+B y-b)-\left(A x^{*}+B y^{*}-b\right)
\end{array}\right)\left(\begin{array}{l}
x-x^{*} \\
y-y^{*} \\
\lambda-\lambda^{*}
\end{array}\right) \\
& =\max \left\{u_{1}^{-1}, u_{2}^{-1}, v_{3}^{-1}\right\}\left[\left(\omega-\omega^{*}\right)^{*}\left(Q(\omega)-Q\left(\omega^{*}\right)\right)\right] \\
& \leq \max \left\{u_{1}^{-1}, u_{2}^{-1}, v_{3}^{-1}\right\} \frac{1}{\beta}\left\{e(x)^{T}\left[\left(\omega-\omega^{*}\right)+\beta\left(Q\left(\omega^{*}\right)-Q(\omega)\right)\right]-e(\omega)^{T} e(\omega)\right\} \\
& \leq \max \left\{u_{1}^{-1}, u_{2}^{-1}, v_{3}^{-1}\right\} \frac{1}{\beta}\left\{\|e(\omega)\|\left(\left\|\omega-\omega^{*}\right\|+\beta\left\|\left(Q\left(\omega^{*}\right)-Q(\omega)\right)\right\|\right)\right\} \\
& \leq \max \left\{u_{1}^{-1}, u_{2}^{-1}, v_{3}^{-1}\right\} \frac{1}{\beta} r(\omega)\left\{\left\|\omega-\omega^{*}\right\|+\beta L\left\|\omega-\omega^{*}\right\|\right\}, \\
& \text { where } v_{3}^{-1}=\| \begin{array}{c}
\left.(A, B)\left(\begin{array}{l}
A^{T} \\
B^{T}
\end{array}\right)\right)^{-1}(A, B) \| \text { Thus, we can conclude that } \\
\left(\begin{array}{l}
(A) \\
(3.6)
\end{array}\right.
\end{array}
\end{aligned}
$$

(3.6) holds.

Theorem 3.2 Suppose that the hypotheses of Lemma 3.2 holds, and $\beta$ satisfies the condition

$$
1>\frac{2 u-\beta}{2 u}-\frac{\beta^{2}}{\max \left\{u_{1}^{-1}, u_{2}^{-1}, v_{3}^{-1}\right\}^{2}\{1+\beta L\}^{2}}>0,
$$

then the sequence $\left\{\omega^{k}\right\}$ converges to a solution of (1.1) $R-$ linearly.

Proof: Combining (3.5) with (3.6), one has

$$
\begin{aligned}
& \frac{\beta^{2}}{\max \left\{u_{1}^{-1}, u_{2}^{-1}, v_{3}^{-1}\right\}^{2}\{1+\beta L\}^{2}}\left\|\omega^{k}-\omega^{*}\right\|^{2} \\
& \leq r\left(\omega^{k}\right)^{2} \leq \frac{2 u-\beta}{2 u}\left\|\omega^{k}-\omega^{*}\right\|^{2}-\frac{2 u-\beta}{2 u}\left\|\omega^{*}-\omega^{k+1}\right\|^{2} .
\end{aligned}
$$

i.e.,

$$
\left\|\omega^{*}-\omega^{k+1}\right\|^{2} \leq\left(\frac{2 u-\beta}{2 u}-\frac{\beta^{2}}{\max \left\{u_{1}^{-1}, u_{2}^{-1}, v_{3}^{-1}\right\}^{2}\{1+\beta L\}^{2}}\right)\left\|\omega^{k}-\omega^{*}\right\|^{2}
$$

By $1>\frac{2 u-\beta}{2 u}-\frac{\beta^{2}}{\max \left\{u_{1}^{-1}, u_{2}^{-1}, v_{3}^{-1}\right\}^{2}\{1+\beta L\}^{2}}>0$, then the desired result follows.

\section{CONCLUSIONS}

In this paper, we proposed a new iterative method for solving the structured variational inequality problem (VI), and have proved its global convergence without carrying out any line search technique. Furthermore, the error bound estimation for VI is also established under the suitable conditions, based on this, we prove that the method has global R-linear convergence rate. Surely, under milder conditions, we may established global error bounds for VI, and may use the error bound estimation to establish quick convergence rate of the method for solving the VI. This is a topic for future research.

\section{ACKNOWLEDGMENT}

The author wish to give their sincere thanks to the editor and the anonymous referees for their valuable suggestions and helpful comments which improved the presentation of the paper.

\section{REFERENCES}

[1] Stadtler H. and Kilger C., Supply chain management and advanced planning. Berlin, Germany: Springer-Verlag, 2002.

[2] Facchinei F.and Pang J.S.,Finite-dimensional Variational Inequality and Complementarity Problems. New York:Springer, 2003.

[3] Wang Y.J., Xiu N.H. and Zhang J.Z.,"Modified extragradient methods for varitional inequalities and verification of solution existence,"J. Optim. Theroy Appl,vol.119,pp. 167-183,2003.

[4] Wang Y.J.," A new projection and contraction method for varitional inequalities," Pure Math. and Appl,vol.13,no.4, pp.483-493,2002.

[5] Gabay D.,"Applications of the method of multipliers to variational inequalities."In:M.Fortin and R.Glowinski(Eds) Augmented Lagrange Methods. Applications to the Solution of Boundary valued Problems(Amsterdam: North Holland), pp.299-331,1983.

[6] Gabay D. and Mercier B.,"A dual algorithm for the solution of nonlinear variational problem svia finite-element approximations,"Computers and Mathematics with Applications ,vol.2, pp.17-40,1976.

[7] Ye C. H. and Yuan X. M.," A descent method for structured monotone variational inequalities," Optimization Methods and Software,vol. 22,no.2, pp.329-338,2007.

[8] Han D.R.,"A modified alternating direction method for variational inequality problems," Applied Mathematics and Optimization,vol. 45, pp.63-74,2002.

[9] Wang Y. J., Xiu N.H., Theory and algorithms for nonlinear programming. Xian: Shanxi science and technology press, pp.171, 2008. (In Chinese)

[10] Noor M.A., "General variational inequalities", Appl. Math. Lett., 1(2), pp. 119-121,1988. 